Meta

Journal des traducteurs

Translators' Journal

\title{
La méthode du discours : quelques outils
}

\section{Jean-Claude Choul}

Volume 24, numéro 3, septembre 1979

URI : https://id.erudit.org/iderudit/002238ar

DOI : https://doi.org/10.7202/002238ar

Aller au sommaire du numéro

Éditeur(s)

Les Presses de l'Université de Montréal

ISSN

0026-0452 (imprimé)

1492-1421 (numérique)

Découvrir la revue

Citer cet article

Choul, J.-C. (1979). La méthode du discours : quelques outils. Meta, 24(3),

336-348. https://doi.org/10.7202/002238ar d'utilisation que vous pouvez consulter en ligne.

https://apropos.erudit.org/fr/usagers/politique-dutilisation/ 


\section{La méthode du discours : quelques outils*}

Le terminologue, selon Henri Dumas (1977), «doit s'occuper de termes » et, ajoute-t-il plus loin, un mot \& vaut ce pour quoi on l'accepte, qu'on nomme pour cette raison son acception ». Nous avons là deux exemples du piège de l'étymologie, signalé par J. Paulhan (1967) et rappelé par J. Rey-Debove (1973). Sans \& faire de l'étymologie » sauvage ou non, il est bon de rappeler que terminologie vient de terminus (mot) et qu'acception signifie sens. La méthode du discours, " infaillible et bien définie [...] n'existe probablement pas », ainsi conclut H. Dumas, se remettant au «bon sens » et à l'« inspiration ». Le traducteur serait-il si démuni? I.V. Spilka (1977) proposait pour sa part un appareil morphosyntaxique entre la paraphrase et la transformation, sans appel apparent au sens. Mais c'est au sens que Dumas laissait le soin de distinguer entre mot et terme.

\section{Mot, terme, expression}

Le mot n'a pas bonne réputation chez les linguistes, et terme se définit par sa fonction dans une phrase ou par les relations qu'il entretient avec d'autres éléments dans une structure, ou encore comme emploi monosémique dans une science (Dubois et al., 1974). Si l'on peut associer mot et dictionnaire dans l'emploi ordinaire, il faut se souvenir que les lexicographes ne connaissent que l'entrée ou l'adresse (J. et Cl. Dubois, 1971).

Qu'advient-il d'expression? Si Dumas cite Littré, il n'est pas déplacé de citer Bréal (1924) : « une langue ne se compose pas uniquement de mots; elle se compose de groupes de mots et de phrases», parmi lesquels il relève les expressions proverbiales, les termes techniques et les phrases plus ou moins consacrées; nous y reviendrons. La première « expression » donnée par Dumas n'est autre que la définition du * mot » cheval, c'est-à-dire sa périphrase (ReyDebove, 1973) ou paraphrase (J. et Cl. Dubois, 1971) qui repose le problème de la synonymie : une librairie est un magasin où l'on vend des livres ou $\mathrm{x}=\mathrm{y}+\mathrm{qui}+$ prédicat. La synonymie est alors à rapprocher de l'hypéronymie

\footnotetext{
Notre titre se veut une paraphrase de celui de $H$. Dumas paru dans Meta, 22-2, juin 1977, p. 110-116: \&La méthode du discours : termes, mots, expressions »: il s'agit ici moins d'une discussion de ses propositions que d'une suggestion d'aborder le pro-
blème par la sémantique.
} 
(relation d'inclusion) : l'âne comme le cheval est un animal, le bouquet comme le fumet est un arôme (cf. Dubois et al., 1971 - D.F.C. ; Rey-Debove, 1973).

Il existe peut-être une autre voie pour aborder le problème du mot, du terme et de l'expression pour le traducteur qui travaille effectivement du discours, mais en relation avec le lexique. C'est pourquoi cheval n'est pas l'exemple idéal, mais plutôt cheval-vapeur (cf. Spilka, 1977) ou quatre chevaux (J. et Cl. Dubois, 1971), c'est-à-dire le syntagme.

\section{Syntagme, synapsie}

H. Dumas (1977) voudrait faire de palan pneumatique à piston un terme constitué de quatre mots (ce terme étant par ailleurs une expression). Mais dans l'opposition terme / mot de Dubois et al. (1974) rayon est un terme s'il entre dans « Rayon $X$ », « rayon gamma et un mot dans \& chef de rayon et "rayon de soleil ${ }^{1}$. On constate que pneumatique prend un sens particulier selon que le terme apparaît dans "palan pneumatique ", "machine pneumatique » ou «monté sur pneumatiques». C'est «dans et par une forme spécifique, celle du syntagme $\gg$ que s'accomplit le sens, selon Benveniste (1976) qui veut rendre au mot son statut d'unité sémantique. Pour identifier les groupes de mots lexicalisés et conserver au syntagme «sa désignation propre qui s'applique à n'importe quel groupement, même occasionnel, opéré par des moyens syntaxiques », Benveniste (1976) a proposé le terme de synapsie, qu'il distingue de la composition (portefeuille, garde-malade), en donnant comme exemples : gardien d'asile, asile de nuit, employé de chemin de fer (où chemin de fer est subsynaptique). Comme pour le terme de Dumas, c'est la désignation, non plus «précise et concise», mais unique et constante qui caractérise la synapsie par opposition au syntagme (chapeau de paille vs le chapeau de ma tante). Benveniste, en proposant la synapsie (dont la liste reste ouverte) faisait allusion aux travaux de L. Guilbert sur le vocabulaire de l'aviation et celui-ci (1975) élargira l'étude du phénomène en proposant l'union syntagmatique ${ }^{2}$, dont il cherchera à retracer la génération (ex : on aménage le territoire $\rightarrow$ le territoire est aménagé $\rightarrow$ l'aménagement du territoire). Le mérite particulier de Guilbert a été de montrer qu'un des critères de Benveniste - l'absence d'article devant le déterminant (le second terme de la synapsie) - n'est ni fondamental ni constamment vérifiable, et d'intégrer sous la nouvelle dénomination des groupes de structure Nom + Adjectif : majorité silencieuse, quotient intellectuel, marée noire, et les «locutions verbales $\$$ : prendre connaissance, prendre la fuite.

Benveniste signalait que le phénomène synaptique caractérisait la nomenclature technique et $P$. Gilbert (1971) indique sa grande fréquence dans le français moderne (il parle de syntagmes qui se lexicalisent et deviennent des unités de sens) : bouche à bouche, compte à rebours, classe de neige, rampe de lancement, coup par coup, perte de vitesse, coussin d'air. On trouvera chez

1. P. Auger et L.-J. Rousseau (1978) parleront de « termes complexes », qui équivaut pour eux à \& syntagme \$: il faut cependant préciser que syntagme est une unité dynamique de travail et non un cas de lexicalisation (unité de rang intermédiaire, cf. Dubois et al., 1974).

2. A. J. Greimas (1972) parle de paralexème et Martinet (1974) a proposé synthème. 
Vigner et Martin (1976) d'excellentes analyses de ce qu'ils appellent les unités lexicales complexes de la langue technique ${ }^{3}$ : vis mère à pas métrique, meule à tronçonner, pompe d'alimentation. Dans ces synapsies, les termes s'articulent au moyen de quatre outils syntaxiques : $d$, de, avec, pour (auxquels on peut ajouter sur) :

ampèremètre avec transformateur de courant ;

abrasif aggloméré pour tonnelage.

Dans les synapsies courantes Benveniste avait déjà cité tailleur pour hommes. On peut ajouter aliments pour chiens, mais on enregistre déjà nourriture de chiens, et pour serait ainsi transitoire. Vigner et Martin ont basé leur étude sur la lexie complexe de B. Pottier (1974).

\section{Lexie}

Avec la notion de lexie (unité lexicale mémorisée), Pottier tente de reprendre le problème à nouveaux frais et de résorber l'apparente discontinuité du phénomène que découpent les mots, les composés (prophète de malheur), les conglomérés (va-nu-pieds, monte-en-l'air), les synapsies (vis à tête fendue), les unités syntagmatiques (machine à calculer, donner lecture), les unités phraséologiques (commettre un crime), les locutions proverbiales (aller a vau-l'eau) et les dictons et proverbes (Après la pluie, le beau temps).

On aura donc (Pottier, 1974) la lexie simple, correspondant au mot traditionnel - unité sémantique de Benveniste —, la lexie composée (tire-bouchons, mais aussi fourchette, remanier - ne s'opposant plus à fourche ou manier), la lexie complexe - en voie de lexicalisation - (guerre froide, feu rouge, prendre des mesures, bel et bien), que Rey-Debove (1973) dit sur la voie de la codification (n'être pas sans savoir, sévère mais juste) et la lexie textuelle, énoncé ou texte. À l'intérieur même de ce découpage, Pottier distingue la lexie invariable (ou figée) qui va de faire flèche de tout bois à on apprend de source géneralement bien informée et la lexie variable, comportant un cadre stable et une zone instable.

$$
\text { Il y a tout lieu de }\left\{\begin{array}{l}
\text { penser } \\
\text { croire } \\
\text { supposer }
\end{array}\right\} \text { que. }
$$

La lexie, toujours selon Pottier, provient d'une habitude associative : en France, avant son envoi en fourrière, la voiture d'un contrevenant est immobilisée à l'aide d'une pince = pince d'immobilisation (sabot de Denver). La «génération 》 de Guilbert n'entre sans doute pas en conflit avec une telle explication. La lexie se propose ainsi de succéder à la locution (que F. Brunot, 1926 (1965) voulait généraliser) où chaque critère de détermination est insuffisant par lui-même. Pottier relève : 1) la non-autonomie d'un composant, avec ou non

3. Désignation due à C. Bally (1951) dont le critère est la cohésion; dans les séries, elle est relative et dans les unités, absolue. Guilbert (1975) rétablit cette classe pour les nonclassables en unités syntagmatiques verbales qui acceptent, elles, les nominalisations. 
possibilité de reprise (le chemin de fer $\rightarrow *$ le chemin), 2) la non-commutabilité d'un composant (se trouver mal $\neq$ bien), 3) la non-séparabilité des composants (*plaque très tournante, mais prendre souvent la mouche), 4) la particularité de structure interne, absence de détermination (perdre courage, mais prendre son temps). On comparera aux critères ci-dessus les six groupes de Spilka (1977). L'habitude de l'association est généralement récusée par les linguistes (critère extra-linguistique), mais leur classement finit par s'en ressentir ${ }^{4}$ : Guilbert la récupère quand ses schémas ne fonctionnent plus, ce qui l'oblige à ouvrir une dernière classe d'unités phraséologiques, considérées comme non productives. On aimerait penser qu'avec le syntagme et la phrase on quitte le domaine du codé pour enfin exercer librement son choix (cf. Rey-Debove, 1973), mais M.-M. Dubois et al. (1973) notent avec justesse qu'il y a des syntagmes rituels que l'on utilise en bloc, au même titre que les lexies (ou les mots). Existe-t-il un moyen de rendre compte de cette interaction du lexique et du discours, de l'existence d'unités lexicales considérées comme du prédiscours?

\section{Collocation}

La collocation permettrait sans doute d'étudier le phénomène (la formation de locutions) dans son ensemble. Réintroduite par Firth (1964) dans sa nouvelle acception, la collocation se présente comme une tendance de cooccurrence, une attente mutuelle et une fréquentation entre les mots que Culioli (1968) définira comme relation formelle fondamentale entre des termes. Elle est à rapprocher du paradigme plutôt que des restrictions de sélection, faisant abstraction des relations ou catégories grammaticales, et recouvre la relation d'association de Pottier (1974) exerçant des contraintes phraséologiques, par laquelle s'établissent des habitudes combinatoires en vertu d'attitudes socioculturelles. La collocation est proprement une combinatoire restreinte, en fonction d'affinités sémantiques et de probabilités culturelles. La collocation ", c'est l'ascenseur / la machine paradigmatique de Galisson (1976) permettant d'obtenir des syntagmes.

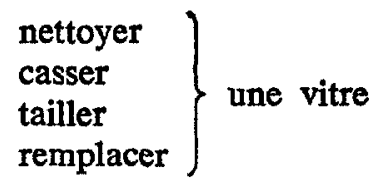

La synapsie puisait ses termes synaptables dans les noms ou verbes à l'infinitif, tandis que la collocation y est indifférente : les collocables s'organisent en familles sémantiques sans contrainte grammaticale stricte. L'exemple d'allusion l'illustre bien :

\begin{tabular}{|c|c|c|}
\hline allusion & $\begin{array}{l}\text { claire } \\
\text { transparente } \\
\text { mystérieuse } \\
\text { personnelle }\end{array}$ & $\begin{array}{l}\text { faire une allusion à qqun / qqch } \\
\text { une allusion échappe à qqun }\end{array}$ \\
\hline
\end{tabular}

4. C'est le cas de Chafe (1968), Weinreich (1969), Fraser (1970).

5. Auger et Rousseau (1978) qui y font allusion - au pluriel - donnent : * eléments lexicaux qui font habituellement partie de (l') environnement (d'un terme) ». 
ou encore de créneau (cf. Gilbert, 1971)

\begin{tabular}{|c|c|c|}
\hline $\begin{array}{l}\text { s'inscrire dans un } \\
\text { avoir son } \\
\text { choisir un } \\
\text { offrir des } \\
\text { ouvrir des }\end{array}$ & créneau(x) & $\begin{array}{l}\text { étroit } \\
\text { ouvert } \\
\text { rentables } \\
\text { d'ajustement }\end{array}$ \\
\hline
\end{tabular}

Où trouve-t-on les collocations? Les dictionnaires unilingues français comme le Petit Robert, le Bordas du français vivant et le Dictionnaire du français contemporain (D.F.C.) s'y réfèrent implicitement pour les sous-entrées ou les «nuances de sens» (les subsenses de Weinreich, 1969). Certains dictionnaires dits «phraséologiques » (U. Lacroix, Dictionnaire des mots et des idées) présentent les familles de collocables les plus fréquentes; il en va de même pour les dictionnaires analogiques (sans cependant les identifier comme telles). La présence de syntagmes et de phrases dans les dictionnaires (D.F.C., Bordas) souligne l'intervention du discours dans le lexique, dont rend compte la collocation. Mais il faut se garder de n'y voir qu'un assemblage, un arrangement moins fortuit qu'un autre.

\section{Transfert, sélection, spécialisation}

Que se passe-t-il dans la collocation ? N'y a-t-il qu'affinité et probabilité d'occurrence? Benveniste (1976) signale que dans la syntagmation de mots, chaque mot ne retient qu'une petite partie de la valeur qu'il a en tant que signe. Good dans good singer, ne veut pas dire charitable ni heavy dans heavy smoker qui souffe d'embonpoint. La syntagmation (ou contextualisation) comporte au moins trois opérations sémantiques liées. La première, le transfert (ou contamination) s'illustre dans passer son permis (de conduire) ; au Xtx ${ }^{e}$ siècle, on parlait de prégnance, le verbe absorbant son complément comme dans un témoin qui dépose, un chrétien qui pratique, plus généralement le déterminé absorbant le sens de son déterminant. Plus intéressantes, la sélection sémantique signalée par Weinreich (1969) et son résultat la spécialisation qui caractériserait, dans son degré le plus élevé, l'idiomaticité. L'autobus et une tasse de café sélectionnent un sens distinct de prendre et le lait et le vent sélectionnent un sens différent de tourner. L'idiomaticité se manifesterait, selon Weinreich, par une double sélection (cosélection), avec une gradation possible dans la spécialisation : prendre le vent (humer), prendre la mer, prendre l'air, prendre le volant (conduire), prendre la voiture (ne pas y aller à pied), prendre la mouche (s'énerver). On observera le même phénomène avec :

$$
\begin{aligned}
& \text { travailler }\left\{\begin{array}{l}
\text { la pâte (la façonner) } \\
\text { le vin (le falsifier) } \\
\text { un cheval (le dresser) } \\
\text { un poisson (le fatiguer) }
\end{array}\right. \\
& \text { vin qui travaille (fermente) }
\end{aligned}
$$

poutre qui travaille (se disjoint et gauchit) 
C'est cette spécialisation sémantique (avec opacité croissante des termes constituants) qui permet de parler de la locution (idiom - Chafe, 1968 ; Weinreich, 1969 ; et Fraser, 1970) comme unité sémantique. Here and there, ici et là ne sont pas une simple combinaison (encore moins addition) de termes, puisqu'ils veulent dire : en divers endroits, il en va de même pour by and large (à tout prendre). Certaines sélections sémantiques ne sont plus «calculables * et doivent être mémorisées comme blocs. C'est le cas de vue de l'esprit, de déjeuner de soleil et de trêve de plaisanteries que «l'essai de traduction » mot à mot que propose $H$. Dumas (1977) classerait comme termes, mais les termes ainsi colloqués (avec une stabilisation remarquable témoignant de leur spécialisation sémantique) demeurent libres d'entrer dans d'autres collocations sans trêve, déjeuner sur l'herbe, prise de vues, l'esprit d'escalier, bain de soleil, plaisanteries de mauvais goût.

\section{Sémiotaxie}

La sélection et par conséquent la spécialisation sémantique se produisent apparemment de façon privilégiée entre verbe et complément (en particulier d'objet direct), sujet et verbe et nom et adjectif. Cette productivité sémantique par la position du mot en syntagme, par ses relations dans le discours, a pu faire dire à P. Guiraud (1970) que l'« origine \& du sens était syntaxique. Pour étudier les différents sens du verbe tirer, il propose un outil : la structure sémiotaxique. A la recherche des «marques du sens », Nida $(1969,1975)$ forge un terme analogue : semotaxis, mais pour lui ce marquage du sens est distinct de celui que réalise la syntaxe (fonction grammaticale du terme) dont voici un exemple : It is a fox, he is a fox, she will fox him; la sémiotaxie est un conditionnement contextuel, fondé sur la comptabilité des catégories sémantiques et leur sélection. Ses exemples de chair comme nom et verbe, de run comme verbe (cinq sens pour le premier et sept pour le second) illustrent bien le phénomène. C'est d'ailleurs ce à quoi en vient Guiraud (1970) avec ses exemples de loup : j'ai abattu un loup, j'ai pêché un loup, elle porte un loup; l'insistance sur la syntaxe s'estompe : il s'agit bien en fait de sélection sémique, comme chez Nida, donc de la syntagmation au sens de Benveniste. Nida (1969) note en fait un recouvrement partiel entre le marquage syntaxique et sémiotaxique, mais que (1975) les classes sémiotaxiques (catégories sémiques) dépassent en nombre et en variété les catégories grammaticales ${ }^{8}$.

S’il est évident que électrique sélectionne un sème (trait sémantique) particulier de chaise et que humain régit électrocuté et non électrifié, le conditionnement contextuel n'en existe pas moins dans des manifestations auxquelles on prête généralement peu d'attention, tant elles sont familières.

Il s'agit des prépositions « obligées » qui sont les premiers éléments contextuels d'un verbe, l'égal en quelque sorte de la détermination du nom et qui constituent le type même de la sémiotaxie. Tout traducteur connaît ces pièges sémanti-

6. On pourra comparer son esquisse de classement (1975) aux restrictions de sélection des générativistes, mais la sémiotaxie comme la collocation, a laquelle il la rattache, sont moins limitatives. 
ques : stand, stand by, stand down, stand for, stand in (for), stand on, stand out, stand to, stand up, stand up for, stand up to. Et où s'insèrent ou s'ajoutent des unités lexicales obligées pour former les locutions : stand (one) in good stead, stand on ceremony, stand to reason, qui illustrent le plus haut degré de spécialisation sémantique. On trouvera dans F. T. Wood $(1974,1975)$ et W. S. Fowler (1976) des répertoires de ces mécanismes encore peu étudiés. Il pourrait sembler que le français n'en possède pas l'équivalent exact. En fait, la sélection par la particule ne paraît pas à première vue être liée à la spécialisation par le complément, c'est-à-dire que la précédence est moins fréquente. Cependant la sélection prépositionnelle existe, que l'on compare payer qqch. et payer de qqch. (avec spécialisation : ne pas payer de mine), se payer qqch. (avec spécialisation : se payer la tête de qqun.) et se payer de qqch. (se payer de mots), ou encore porter qqch., porter qqun. à qqch., porter sur qqch. (spécialisation : porter sur les nerfs de qqun.). D'ailleurs, l'anglais ne passe pas nécessairement par la particule pour la sélection (la sélection est alors combinée à la spécialisation) : eat crow, call names, call a halt, boss the show, evade the issue. II faut aussi noter que l'absence de particule ou de complément sélectionne un sens - l'intransitif, l'emploi intransitif (construction absolue). Ex. : boucler, frayer, bouger, nicher, ressusciter, etc. On consultera les répertoires de E. Lasserre (1965), J.P. et J. Caput (1976) et M.-M. Dubois et al. (1973), ce dernier ouvrage se consacrant aux « syntagmes rituels» et comportant la traduction anglaise des «locutions».

\section{Paramètre}

On n'a pas abordé la question de l'emploi figuré, en raison de son manque de pertinence : l'emploi figuré n'est pas \&le propre » de la locution et constitue seulement un type de spécialisation sémantique issue de collocations de faible fréquence ; il suffit de feuilleter le Petit Robert pour s'en convaincre (cf. Guiraud, 1970). Il demeure cependant nécessaire d'introduire une dernière notion qui permettrait de rendre compte de la locution et de prendre le relais de la sémiotaxie arrivée à la spécialisation. Cette dernière ne doit pas être assimilée au «figement $\gg:$ une spécialisation sémantique peut être le propre d'une seule occurrence. C'est la constance de désignation que Benveniste attribue aux synapsies qui permet de marquer le passage de la spécialisation au paramètre. Cette constance peut avoir pour objet tant une réalité physique qu'une attitude ou un comportement. Elle est donc essentiellement reproductible et peut constituer une constante du discours (spécialisation sémantique devenue unité).

La notion de paramètre est proposée par l'écrivain Jean Paulhan (1966) comme nouvelle figure, paradoxalement conventionnelle et productive, pour prendre en charge «la citation, l'expression toute faite, la locution proverbiale, le proverbe ». Il importe ici de se garder des jugements de valeur, c'est pourquoi paramètre peut se révéler utile comme instrument. Si le rédacteur doit s'« inspirer des bons auteurs, selon H. Dumas (1977), se renouveler et oublier les expressions toutes faites, les locutions usées, les clichés banals », le traducteur, intermédiaire dans une communication, doit viser à la lisibilité et assumer les contraintes de la conventionnalité du langage, en particulier dans les sciences et les techniques. 
On a vu qu'il ne suffit pas que les mots constituant une expression soient compréhensibles pour que passe le sens. Un traducteur doit être conscient des collocations, des mécanismes de la sémiotaxie et acquérir une sensibilité aux paramètres. Comment pourrait-il, s'il en était autrement, traduire to go out of one's way? A vouloir sortir des chemins battus on risque de battre la campagne, pourrait-on dire. Ce qui nous renvoie au problème de l'attestabilité.

\section{Attestabilité}

Pour les « expressions 》 et les « tournures » au sens de Dumas, c'est-à-dire les collocations et les paramètres, on pourra se reporter aux dictionnaires de phrases comme le D.F.C. et le Bordas, celui-ci affichant 11000 locutions pour 34000 mots, et bien sûr le Petit Robert qui, si toutes les locutions y faisaient l'objet d'entrées distinctes, compterait 250000 entrées.

Le cas des termes techniques et du palan pneumatique à piston (air-cylinder hoist) posent à la fois le problème de l'attestabilité et de la constance de désignation (paramètre) qui est censée caractériser les synapsies, et l'on peut se demander si la collocation et la sémiotaxie sont utiles là où il s'agit de : nommer » des objets (c'est-à-dire où la description encyclopédique empiète sur la définition). Un rapide examen des principales collocations permet de voir quelles sont les contraintes :

1

palan $\left\{\begin{array}{l}\text { à vis } \\ \text { à chaîne } \\ \text { pneumatique } \\ \text { électrique } \\ \text { monorail }\end{array}\right.$

aussi : transporteur aérien à monorail
2

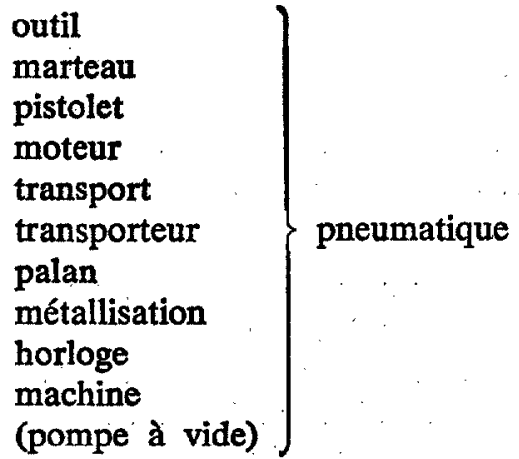

aussi : moteur à air comprimé
3

compresseur à piston

( $\neq$ centrifuge, axial)

pompe à piston

alternatif

$(\neq$ rotative, etc.)

moteur pneumatique

à piston

$(\neq$ à palettes, etc.)

$\left.\begin{array}{l}\text { aussi : } \begin{array}{l}\text { cornet } \\ \text { fusil }\end{array}\end{array}\right\}$ à piston

Et une brève incursion dans quelques répertoires techniques ?, limitée aux palans, donne :

air hoist

$$
\left\{\begin{array}{l}
\text { treuil à air comprime } \\
\text { appareil de levage pneumatique } \\
\text { treuil pneumatique }
\end{array}\right.
$$

7. Consultés pour les collocations : Petit Larousse 1975, Petit Robert 1970, Larousse 3 vol. 1965, Quillet-Flammarion 1963, D.F.C. 1971, Bordas 1976, Alpha Encyclopédie 1968, et pour les traductions relatives au palan pneumatique : F. Cusset, Vocabulaire technique, 1977, G. Malgorn, Dictionnaire technique, 1972, Auger et Rousseau, Lexique A...F. de l'industrie minière, 1973. 


$$
\begin{array}{ll}
\left.\begin{array}{l}
\text { air motor hoist } \\
\text { pneumatique hoist }
\end{array}\right\} \text { palan à air comprimé } \\
\text { air tugger } & \left\{\begin{array}{l}
\text { treuil à air comprimé } \\
\text { treuil pneumatique }
\end{array}\right.
\end{array}
$$

Benveniste (1976) signale trois rôles de da dans la synapsie : destination (brosse à habit), caractéristique distinctive (bête d cornes), agent moteur (moulin à vent) - mais ajoute que seule la nature du désigné permet de distinguer celui dont il s'agit : ex. : moulin à café $\neq$ moulin à vent. Dans les collocables ci-dessus, pneumatique est l'équivalent sémantique de à air comprimé, construit sur le modèle sémique de à chaîne - dont le sème dominant est « agent moteur ». Cette remarque n'est pas valable pour aérien, ou à monorail, et non plus pour à piston, où le sème sélectionné est «caractéristique distinctive ». Le moteur pneumatique ou à air comprimé est actionné par l'air comprimé, et si on lui adjoint $a$ piston c'est pour le distinguer des autres moteurs pneumatiques. En outre, selon la définition de Potts cité par Dumas (1977), le piston est un intermédiaire entre l'agent moteur et l'outil, comme dans le marteau pneumatique, donc l'air est comprimé avant d'arriver au piston (le rapport est inverse dans le compresseur à piston). Il semble donc possible d'impliciter à piston, puisque celui-ci est présupposé par la désignation, hormis les cas où le palan serait actionné par l'intermédiaire d'un moteur pneumatique à palettes, mais la difficulté pourrait être tournée en proposant palan à piston, où pneumatique (l'agent moteur) est alors présupposé.

Si la sémiotaxie a permis d'analyser le mécanisme de constitution de la synapsie, la consultation des sources bilingues ne résoud pas la question du paramètre, car si appareil de levage est l'hypéronyme de treuil et de palan, ceuxci ne sont pas synonymes. À première vue, le paramètre (désignation constante) ne semble pas exister.

Le problème peut également être illustré par une réalisation technologique relativement récente. Si l'on avait à traduire air cushion vehicle au moyen des sources courantes, on aurait à choisir entre véhicule à / sur coussin d'air, appareil d coussin d'air, en se gardant d'y ajouter aéroglisseur (hovercraft) qui n'est qu'un hyponyme avec aérotrain. Certains voudraient réserver appareil à coussin d'air pour les engins qui utilisent l'effet de sol, sans être des véhicules à proprement parler, mais comment rendra-t-on surface skimmers qui est le générique (hypéronyme de hovercraft et hydrofoil (hydroptère) ? Si les paramètres se sont formés en anglais, il ne semble pas en aller de même en français.

I. V. Spilka (1977) aborde indirectement le cas des synapsies (donc de collocation et de la sémiotaxie) avec work ethic = éthique du / de travail, et met au point un appareil syntaxique qui assure certaines manipulations sans faire appel au sens, mais lorsqu'il s'agira de préférer l'une des deux versions c'est sur deux définitions (donc sur le sens) qu'elle s'appuiera. Ici encore se pose le problème de l'attestabilité et corollairement de la collocabilité. Éthique de travail serait, selon elle, un système de règles morales issu de la Réforme 
donnant aux vertus du travail la prééminence, tandis que éthique du travail concernerait les règles d'un travail particulier ou, mieux, d'un groupe de travailleurs, avec comme variante éthique de la profession, qu'elle dit équivalente de déontologie. $\mathrm{Du}$ correspond ainsi à de la et renvoie à particulier.

Un moyen de vérifier la plausibilité de la solution offerte consiste à relever les collocations d'éthique d'une part et de travail de l'autre. Si en anglais sont attestés Christian ethics, zoological ethics, sea ethics, church ethics, medical ethics, le français ne présente en regard qu'éthique protestante, éthique chrétienne, éthique bourgeoise, éthique puritaine, éthique de conviction, éthique de responsabilité $^{8}$. Pour travail, la liste des collocables peut se subdiviser en deux séries ${ }^{\circ}$ :

\begin{tabular}{|c|c|c|}
\hline \begin{tabular}{l} 
accident \\
cabinet \\
table \\
séance \\
langue \\
méthode \\
plan \\
lieu \\
bleu \\
instruments \\
compagnons \\
convention \\
\multicolumn{1}{c}{ collective } \\
contrat \\
unité \\
arrêt \\
certificat \\
interruption \\
heure
\end{tabular} & de travail & $\begin{array}{l}\text { accident } \\
\text { Bourse } \\
\text { législation } \\
\text { droit } \\
\text { code } \\
\text { conflit } \\
\text { inspection } \\
\text { inspecteur } \\
\text { médecine } \\
\text { division } \\
\text { monde } \\
\text { ministère } \\
\text { organisation } \\
\quad \text { internationale } \\
\text { médaille d'honneur } \\
\text { ordre du mérite } \\
\text { organisation } \\
\quad \text { scientifique } \\
\text { confédération } \\
\text { générale } \\
\text { cessation concertée } \\
\text { analyse } \\
\text { mesure }\end{array}$ \\
\hline
\end{tabular}

Accident est le seul à apparaître dans les deux séries. Doit-on y admettre éthique? Sur quel principe? Benveniste (1976) signale table de travail parmi les exemples où le joncteur de indique "la circonstance à laquelle l'objet est approprié »; ce sème peut être étendu à la majorité des cas de la série de gauche. Si éthique doit y figurer, éthique de travail comportera donc un sème s circonstance », ce qui va à l'encontre de la définition proposée par I. V. Spilka

8. Ces deux dernières sont relevées chez J. Freund * Max Weber », Encyclopaedia Universalis, 1973, mais E. Weil à l'article * Morale \$, à propos de Weber, parlera de morale de la fidélité aux principes (conviction), morale de la responsabilité quant aux conséquences. Sauf erreur, éthique n'est pas attesté aux articles * Déontologie * et \& Droit médical de l'E.U. Les collocations anglaises sont tirées du Shorter Oxford, 1973.

9. Le relevé s'est fait sur le D.F.C., Bordas, Petit Robert, Larousse 3 vol. 
pour cette synapsie. Il n'y aurait donc qu'une synapsie possible : éthique du travail. I. V. Spilka proposait au début de son article l'analogie :

pleasure principle $=$ principe de plaisir

work ethic $=\mathrm{x}$

Mais outre le fait que principe de plaisir appartient à un système conceptuel particulier (où l'on note au passage : principe de réalité, principe de constance, compulsion de répétition, représentation de but), principe de plaisir comporte un sème «but ${ }^{10} »$, tandis qu'une éventuelle éthique de / du travail comporterait un sème "source », de respectabilité par exemple (cf. la définition de Spilka). Et si éthique comporte un sème "règles », la synapsie prendra la forme d'éthique du travail, mais la collocabilité d'éthique semble s'y refuser : et morale exclurait d'emblée de :

morale du travail

*morale de travail

on comparera aussi :

normes du travail vertus du travail

normes de travail *vertus de travail

Enfin, si work ethic a une spécificité historique ou institutionnelle, comme l'O.I.T., le monde du travail ou la division du travail, c'est encore du qui l'emporterait.

\section{Collocation, sémiotaxie, paramètre}

Il n'a pu s'agir ici que d'esquisser à grands traits les possibilités de ces trois notions. Elles ne doivent pas servir à étiqueter ou à fonder une nouvelle taxinomie : ce sont avant tout des outils qui pourraient permettre de rendre compte de la production du sens dans le discours et, ainsi, servir au traducteur. Elles se veulent pratiques et pragmatiques. Si la collocation a déjà fait l'objet de recherches, il n'en va pas de même pour la sémiotaxie et le paramètre, présentés ici comme ses corollaires en discours : la sémiotaxie pose la nécessité d'étudier la structure sémique d'un terme dans ses relations avec d'autres termes, c'est-à-dire dans ses réalisations par stabilisation de collocables, transfert, sélection et spécialisation sémantiques, et le paramètre prend le relais pour décrire et étudier les réalisations canoniques (synapsies, lexies, locutions). Par souci d'éviter une discussion trop technique, un certain nombre d'hypothèses sous-jacentes n'ont pas été exposées, il semble pourtant nécessaire en terminant, de les évoquer brièvement; il s'agit de la compatibilité sémantique qui serait fondée sur la redondance sémique : les collocables se réunissent parce qu'ils partagent un sème au moins du processus de sémantisation par sémiotaxie; acquisition de sèmes en contexte, et de la formation des paramètres par affinité sémantique et contraintes extra-linguistiques : réalisations technologiques, événe-

10. G. P. Brabant, Clefs pour la psychanalyse, Seghers, 1973, \& l'ensemble de l'activité psychique a pour but la recherche du plaisir et l'évitement du déplaisir $\star$. On notera que s'il y a analogie, elle doit se faire à partir de l'allemand et non entre le français et
l'anglais. 
ments sociaux, standardisations des conditions de vie, modes de toutes sortes et contraintes propres à la communication. On n'a pas non plus abordé le mode d'organisation interne et externe des familles de collocables, mais on peut songer que l'hypéronymie n'y est pas étrangère.

Il est clair que ces notions ne sont pas « achevées » et qu'elles demeurent tributaires de leur objet, le discours, mais elles offrent l'avantage de ne pas ramener de force à la syntaxe ou à une formalisation prématurée des phénomènes qui y échappent. Ce sont des «notions de travail» qui ne prouveront leur cohérence et leur rentabilité que dans les applications, que l'on souhaitera nombreuses.

Jean-Claude Choul

\section{BIBLIOGRAPHIE}

AUGER, P. et L.-J. ROUSSEAU, 1973, Lexique anglais-français de lindustrie minière, Québec, Office de la langue française, 91 p.

- 1978, Méthodologie de la recherche terminologique, Québec, O.L.F., 80 p.

BALLY, C., 1951, Traité de stylistique française, 3e éd., Paris,, Klincksieck, vol. 1, 331 p. BENVENISTE, E., 1976, Problèmes de linguistique générale II. Paris, Gallimard, 286 p. BREAL, M., 1924, Essai de sémantique, science des significations, Paris, Hachette, 372 p.

BRUNOT, F., 1965, Méthode, principes et plan d'une théorie nouvelle du langage appliquée au français, Paris, Masson et Cie, 982 p., $3 \mathrm{e}$ éd. rev., 3 e tirage.

CAPUT, J. et J.P., 1976, Dictionnaire des verbes franģais, Paris, Larousse, 589 p.

CHAFE, W.L., 1968, \& Idiomaticity as an anomaly in the Chomskyan paradigm $\gg$, Foundations of Language, Vol. 4, No. 2, Dordrecht, D. Reidel, p. 109-127.

CULIOLI, A., 1968, × Concepts linguistiques », Alpha Encyclopédie, Paris, La Grange, Batelière.

DUBOIS, J. et C., 1971, Introduction a la lexicographie : le dictionnaire, Paris, Larousse, 217 p.

DUBOIS, J. et al., 1974, Dictionnaire de linguistique, Paris, Larousse, 516 p.

DUBOIS, M.-M. et al., 1973, Dictionnaire français-anglais de locutions et expressions verbales, Paris, Larousse, 386 p.

DUMAS, H., 1977, \& La méthode du disours : termes, mots, expressions », Meta, 22-2, juin 1977, p. $110-116$.

FIRTH, J.R., 1964, Papers in Linguistics, 1934-1951, Londres, Oxford University Press, $233 \mathrm{p}$.

FOWLER, W.S., 1976, Dictionary of Idioms, London, Nelson, 103 p.

FRASER, B., 1970, « Idioms within a transformational grammar », Foundations of Language, Vol. 6, No. 1, Dordrecht, D. Reidel, p. 22-42.

GALISSON, R., 1976, Inventaire thématique et syntagmatique du français fondamental, Paris, Hachette/Larousse, 82 p.

GILBERT, P., 1971, Dictionnaire des mots nouveaux, Paris, Hachette/Tchou, 572 p.

GREIMAS, A.J., 1972, Sémantique structurale, Paris, Larousse, 262 p.

GUIIBERT, L., 1975, la Créativité lexicale, Paris, Larousse, 285 p.

GUIRAUD, P., 1970, Structures étymologiques du lexique français, Paris, Larousse, 211 p.

LASSERRE, E., 1965, Est-ce à ou de ?, Lausanne, Payot, vol. I, 63 p. 
MARTINET, A., 1974, Eléments de linguistique générale, Paris, Colin, 221 p.

NIDA, E.A., 1975, Language Structure and Translation, Stanford, Stanford University Press, 284 p.

NIDA, E.A. et C.R. TABER, 1969, The Theory and Practice of Translation, Leyde, E.J. Brill, 220 p.

PAULHAN, J., 1966, Traité des figures, in J. Paulhan, Euvres complètes, Paris, Cercle du livre précieux, t. II, p. 195-237.

- 1967, Alain, ou la preuve par l'étymologie, in J. Paulhan, Euvres complètes, Paris, Cercle du livre précieux, , t. III, p. 263-286.

POTTIER, B., 1974, Linguistique générale, théorie et description, Paris, Klincksieck, 338 p.

REY-DEBOVE, J., 1973, «Lexique et dictionnaire *, in B. Pottier (dir.), Le langage, Paris, C.E.P.L., p. $82-109$.

SPILKA, I.V., 1977, « Translation of Binominal Phrases 》, Meta 22-3, septembre 1977, p. 171-183.

VIGNER, G. et A. MARTIN, 1976, le Français technique, Paris, Hachette/Larousse, 111 p.

WEINREICH, U., 1969, \& Problems in the Analysis of Idioms », in J. Puhvel (éd.), Substance and Structure of Language, Berkeley, University of Calif. Press, p. 23-81.

WOOD, F. T., 1974, English Verbal Idioms, London, The MacMillan Press, 325 p.

- 1975, English Prepositional Idioms, London, The MacMillan Press, 562 p. 